\title{
The effect of a magnetic field on the radiative excitation and the damping of p-modes
}

\author{
Hideyuki Saio \\ Astronomical Institute, Graduate School of Science, Tohoku University, Sendai 980-8578, \\ Japan \\ e-mail: saio@astr.tohoku.ac.jp
}

\begin{abstract}
A rapidly oscillating Ap star pulsates in high-order p-modes under the influence of a strong magnetic field. The strong field distorts spatially the angluar and the radial pulsation amplitude (eigenfunction). To study the effect of the magnetic field on the radiative excitation and the damping of p-modes, we performed a fully nonadiabatic analysis including the effect of a dipole magnetic field. A magnetic field always tends to stabilize low order p-modes. In the presence of a magnetic field stronger than $\sim 1 \mathrm{kG}$, all $\delta$ Scuti type pulsations are stabilized. For high-order p-modes, on the other hand, the magnetic field enhances $\kappa$-mechanism excitation in some range of the field strength, depending on the pulsation frequency.
\end{abstract}

Keywords. Stars: early-type, stars: chemically peculiar,stars: magnetic fields, stars: oscillations,stars: variables: other

\section{Introduction}

Rapidly oscillating Ap (roAp) stars show light variations with periods between about 6 and 15 minutes (between 2.8 and $1.1 \mathrm{mHz}$ in frequency). These variations arise from high order p-modes modified by a strong magnetic field. This paper shows preliminary results of a nonadiabatic analysis for magnetically modified p-mode pulsations.

We have applied our analysis to an unperturbed model of a $1.9 M_{\odot}$ Main-Sequence star with a composition of $(X, Z)=(0.7,0.02)$, in which envelope convection is suppressed. The model's parameters are $\log L=1.164, \quad \log T_{\text {eff }}=3.9125$, and $\quad \log R=0.281$.

We assume that the star has a dipole magnetic field:

$$
\boldsymbol{B}_{0}=\frac{B_{p}}{(r / R)^{3}}\left(\boldsymbol{e}_{r} \cos \theta+\boldsymbol{e}_{\theta} \frac{1}{2} \sin \theta\right) .
$$

We have expanded displacement vector $\boldsymbol{\xi}$, and the Eulerian perturbation of magnetic field $\boldsymbol{B}^{\prime}$ as

$$
\boldsymbol{\xi}=e^{i \sigma t} \sum_{\ell}\left(\xi_{r}^{\ell} Y_{\ell}^{0} \boldsymbol{e}_{r}+\xi_{\mathrm{p}}^{\ell} \frac{d Y_{\ell}^{0}}{d \theta} \boldsymbol{e}_{\theta}\right), \quad \boldsymbol{B}^{\prime}=e^{i \sigma t} \sum_{\ell}\left(b_{r}^{\ell} Y_{\ell}^{0} \boldsymbol{e}_{r}+b_{\mathrm{p}}^{\ell} \frac{d Y_{\ell}^{0}}{d \theta} \boldsymbol{e}_{\theta}\right)
$$

where $Y_{\ell}^{0}$ stands for sherical harmonics with $m=0$. The scalar variables such as $p^{\prime}$, $\delta L_{\mathrm{rad}}$, and $\delta s$ are expanded as, e.g.,

$$
p^{\prime}=e^{i \sigma t} \sum_{\ell} p_{\ell}^{\prime} Y_{\ell}^{0}
$$

The method of computation is similar to that in the adiabatic analysis of Saio \& Gautschy (2004) but here we have included the entropy and the luminosity perturbations. 


\section{Results}

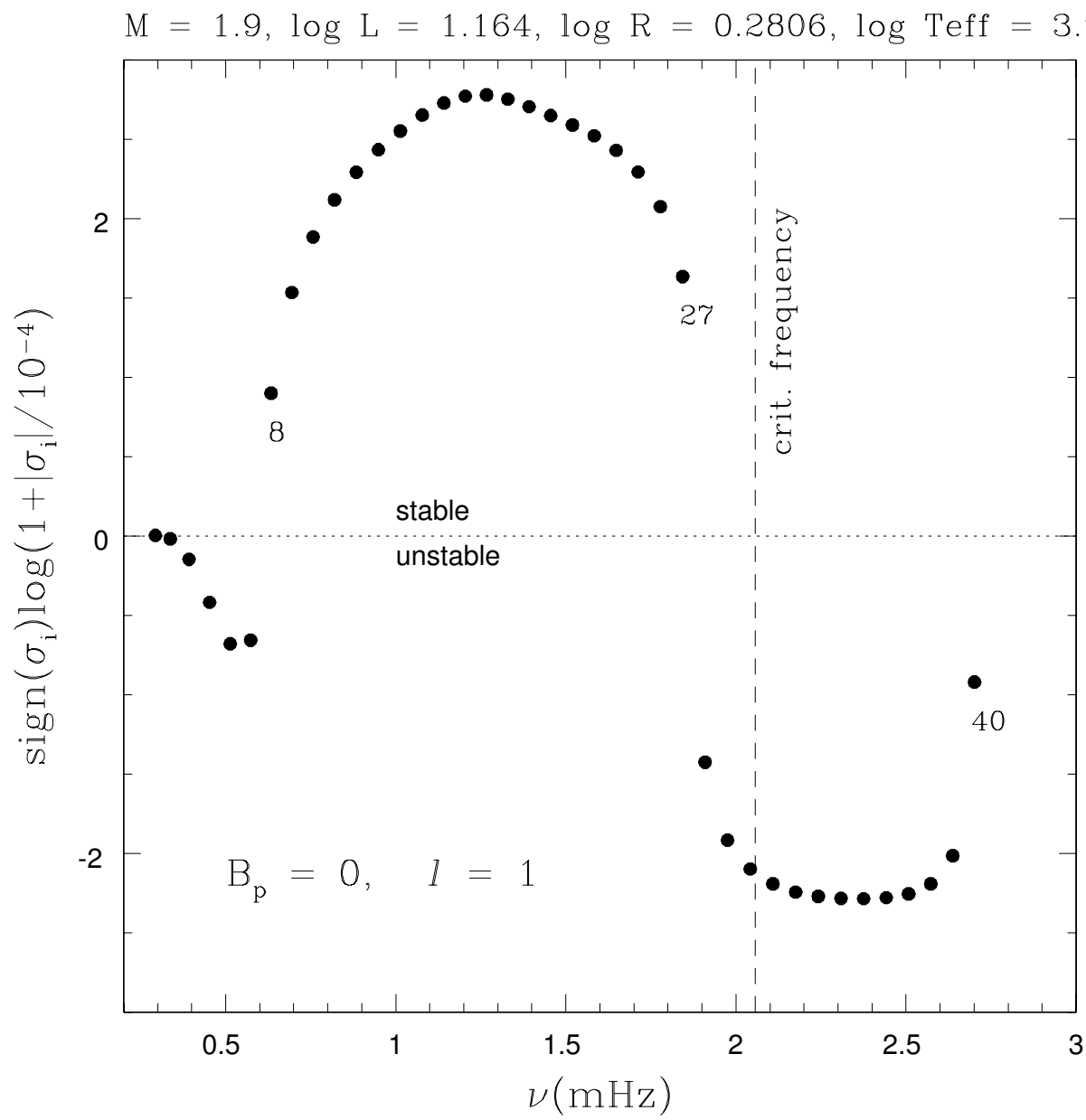

Figure 1. Stability of pulsation modes in the absence of a magnetic field. Pulsation frequency versus damping rate for the 2 nd to the 40 th order $\ell=1 \mathrm{p}$-modes in the absence of magnetic field. The $\kappa$-mechanism in the $\mathrm{He}^{+}$ionization zone excites low order (3rd to 7 th) p-modes, while the $\kappa$-mechanism in the H-izonization zone excites three (28th-30th) high order-modes below the critical frequency. 


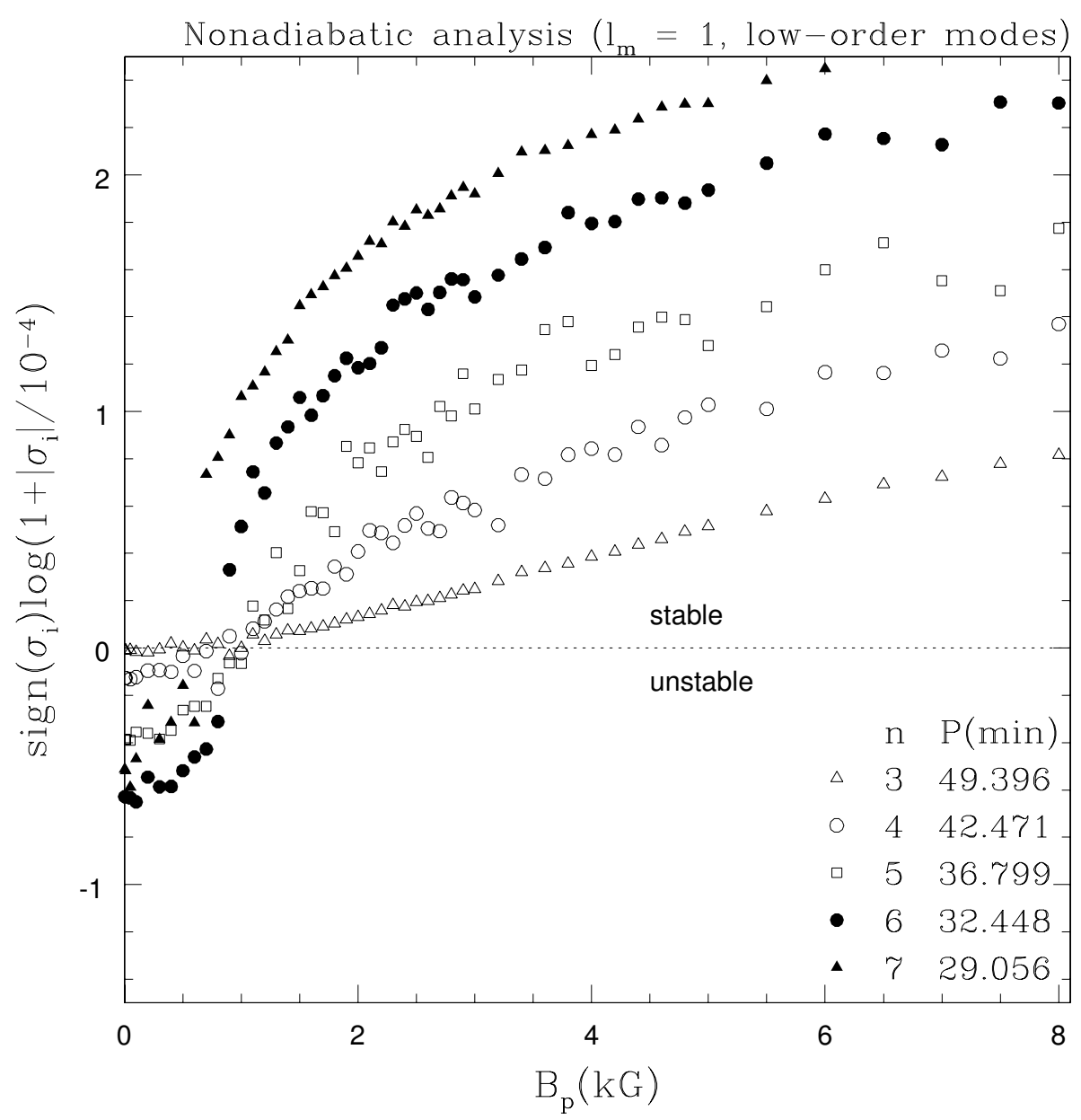

Figure 2. Magnetic damping in low-order modes. Damping rate versus the strength of magnetic fields for the low-order modes which are excited in the absence of magnetic field. Due to the magnetic damping caused by slow waves, all the $\delta$ Scuti type pulsations are suppressed if $B_{\mathrm{p}}$ is larger than $\sim 1 \mathrm{kG}$. 


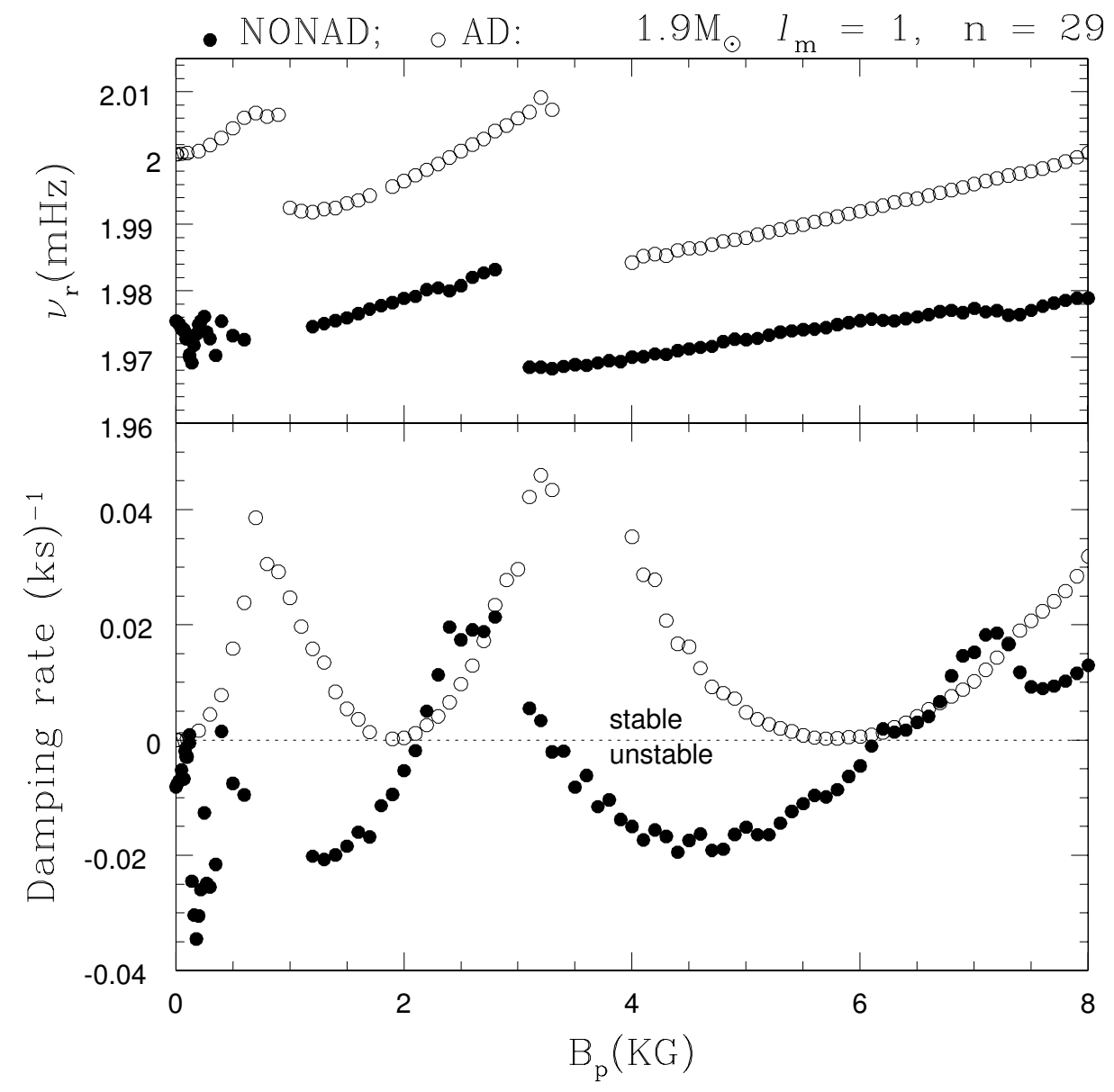

Figure 3. Comparison adiabatic vs nonadiabatic high-order modes I. Pulsation frequency (upper panel) and damping rate (lower panel) as functions of $B_{\mathrm{p}}$ for the 29th order p-mode of $\ell_{m}=1$. Filled and open circles show data from nonadiabatic and adiabatic analyses, respectively. Compared with the adiabatic situation, the frequency jumps (damping-rate peaks) lie at different field strengths in the nonadiabatic case. This mode is unstable for $3.5<B_{\mathrm{p}}(\mathrm{kG})<6$ and $B_{\mathrm{p}}(\mathrm{kG})<2$. 


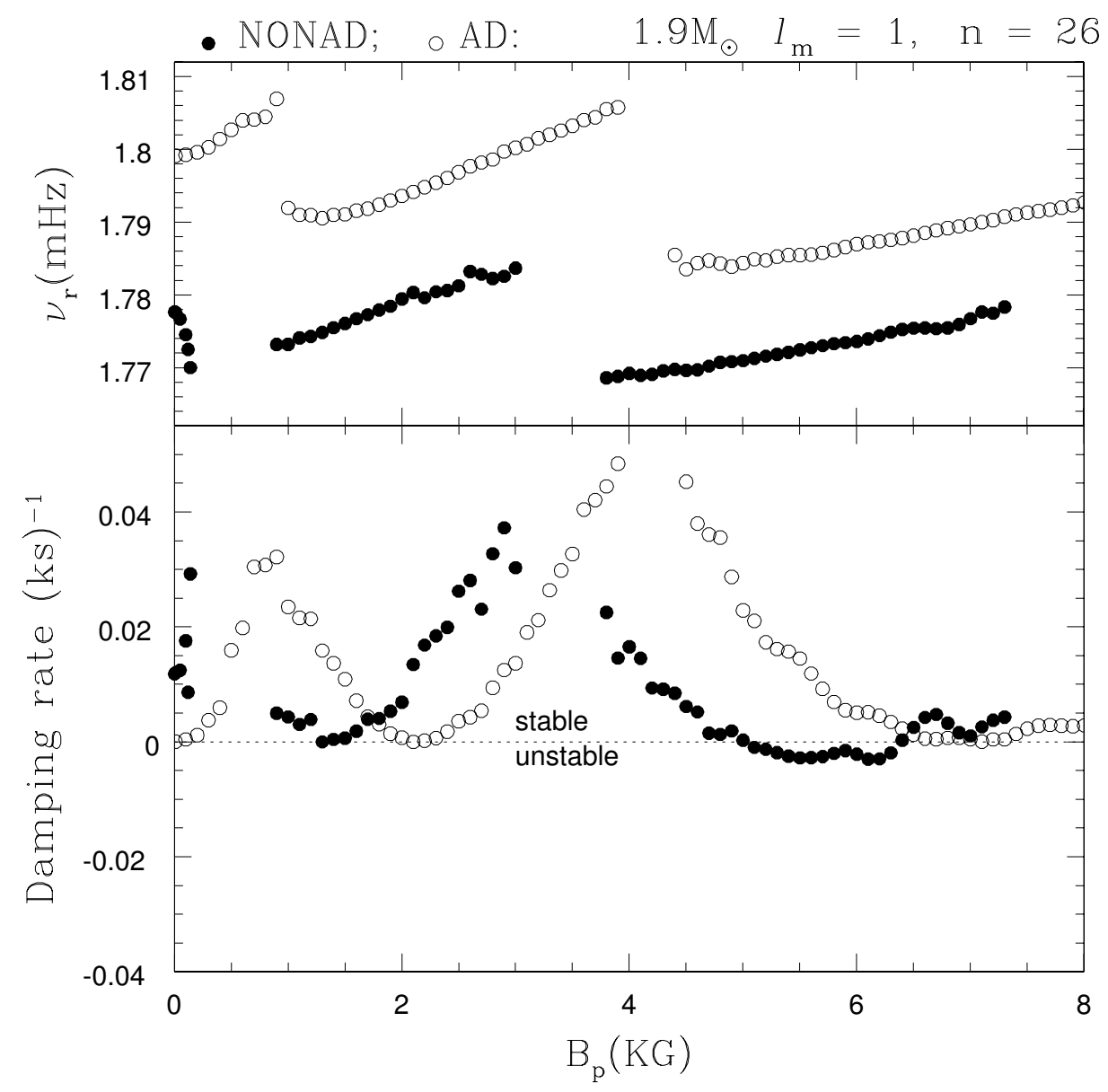

Figure 4. Comparison adiabatic vs nonadiabatic high-order modes II. The same as the previous figure but for the 26 th p-mode. This mode is stable at $B_{p}=0$, but becomes marginally unstable in a range of $5<B_{\mathrm{p}}(\mathrm{kG})<6.5$.

\section{References}

Saio, H. \& Gaurschy, S. 2004, MNRAS 350, 485 